\title{
Nuevas Tecnologías inalámbricas en la Proyección de contenidos en las aulas mediante la utilización de dispositivos móviles.
}

\author{
Héctor Molina García \\ Universidad Francisco de Vitoria \\ h.molina@ufv.es
}

\begin{abstract}
Resumen:
La transmisión de conocimientos en las aulas cuenta con nuevas herramientas tecnológicas que facilitan en gran medida el objetivo buscado, este es el caso de la proyección de contenidos utilizando dispositivos móviles de forma inalámbrica. Profesores y alumnos pueden transmitir contenidos a un proyector de forma inalámbrica y con una calidad similar a la obtenida utilizando la transferencia mediante cable.
\end{abstract}

Palabras clave: Tecnología educativa; nuevas tecnologías; proyección de contenidos; dispositivos móviles; tecnología s inalámbricas.

\section{New Wireless Technologies in the classroom content's design by using mo- bile devices.}

\begin{abstract}
:
The transmission of knowledge in the classrooms has new technological tools that facilitate in a good way the desired goal, this is the case of the projection of content by using mobile devices wirelessly. Teachers and students can stream contents to a projector wirelessly and with similar quality to the one obtained by using the cable transfer way.
\end{abstract}

Key Words: Educational technology, New Technologies, Content Projection, Mobile Devices, Wireless Technologies.

\section{Referencia normalizada:}

Molina García, H. (2014): Nuevas tecnologías inalámbricas en la proyección de contenidos en las aulas mediante la utilización de dispositivos móviles. Historia y Comunicación Social. Vol. 19. Núm. Especial Febrero. Págs. 261-272.

Sumario: 1. Introducción. 2. Metodología. 3. Nuevas Tecnologías en educación. 4. Los dispositivos móviles como fuente en la proyección de contenidos en las aulas. 4.1. Una breve visión histórica del proyector y la integración de sus diferentes tecnologías. 4.2. El proyector como herramienta fundamental en los procesos educativos. 4.3. Posibilidades técnicas en para la proyección de contenidos mediante dispositivos móviles en las aulas. 5. Conclusiones. 6. Bibliografía.

\section{Introducción}

En la actualidad las nuevas tecnologías móviles conquistan todos los campos de nuestra sociedad, todos los usuarios de estas tecnologías tratan de adaptarlas a sus 
trabajos y a su vida personal. Este es caso de la transmisión de conocimientos en las aulas. Alumnos y profesores utilizan nuevas herramientas, tabletas y smartphones y sus innumerables aplicaciones para la adquisición de conocimientos y para facilitar el trabajo dentro de las aulas.

Este proceso de innovación y adaptación, al contrario que la mayoría de herramientas didácticas, ha surgido desde abajo, has sido los alumnos los encargados de encabezar esta transformación. Los jóvenes estudiantes, más acostumbrados en el manejo de las nuevas tecnologías móviles, han tratado de utilizarlas en las clases para comunicarse, para la transferencia de archivos, para la toma de apuntes, etc. Por su parte, los profesores se han percatado de este hecho y han intentado ponerlo de su lado estudiando las distintas aplicaciones que se pueden hacer con ellos.

En este sentido, la aparición de nuevas tecnologías móviles aplicadas a las aulas desplazarán a las tecnologías ya obsoletas, y las que no logren adaptarse o encontrar hueco serán mártires tecnológicos, como fue el caso, por ejemplo de los retroproyectores o de las pizarras de tiza.

Profesores y alumnos son conocedores del potencial que llevan en sus bolsillos, en sus carteras y en sus mochilas. Tienen potentes herramientas informáticas con conexiones a internet de banda ancha inalámbricas desde las que poder consultar apuntes, asistir a clases virtuales, descargar prácticas, trabajar en ellas, ingresar en aplicaciones desarrolladas a medida, etc. La venta de dispositivos móviles de última generación, tabletas y smartphones, ha crecido exponencialmente en los últimos años, esto hace que el futuro sea más claro y evidencia el camino por el que transcurrirán los próximos desarrollos.

Desde la aparición de la linterna mágica, pasando por los retroproyectores, muy utilizados en las aulas, se ha llegado a la tecnología de proyección actual, que permite proyectar cualquier tipo de contenido: imágenes, presentaciones, vídeos, etc, incluso vídeos en tres dimensiones o con calidades en alta definición. Hasta ahora esta tecnología estaba disponible mediante la utilización de un cable que transfería el contenido a los proyectores.

Este hecho está cambiando, ya que actualmente existen tecnologías que permiten la proyección de contenidos de forma inalámbrica con una calidad y estabilidad aceptables, como puede ser la tecnología Airplay de Apple o la tecnología DLNA. Estos avances tecnológicos pueden ser aprovechados por los actores que intervienen en el proceso formativo, para que sean nuevas herramientas que ayuden en la transferencia de conocimientos y colaboren en la comprensión de contenidos didácticos.

\section{Metodología}

Esta investigación no intenta esclarecer los paradigmas relativos a las nuevas herramientas y aplicaciones móviles de carácter docente, fundamentalmente trata 
de investigar los nuevos recursos docentes para la proyección de contenidos en las aulas utilizando como fuente los dispositivos móviles, centrándose en las tecnologías inalámbricas. En la actualidad cohabitan numerosas tecnologías, sistemas operativos, diferentes modelos y marcas que imposibilitan y dificultan la lógica estandarización en este sentido.

Por un lado, los recursos económicos marcan el camino necesario que hay que seguir, ya que la inversión en nuevas tecnologías en la proyección de contenidos con fuentes móviles inalámbricas es arriesgada, aún es muy complicado descifrar el futuro en este sentido, y una fuerte inversión hacia una tecnología no adecuada puede traer problemas.

Por otro lado, las necesidades tecnológicas docentes también intervienen en la tendencia tecnológica, la demanda de estos nuevos recursos debe partir de los actores que intervienen en el proceso formativo, ya que una iniciativa que no se inicie desde estos actores corre el riego de no ser utilizada, y por lo tanto, condenada al ostracismo.

En función de las necesidades de las asignaturas y de alumnos y profesores y de los recursos económicos de los que se disponga existen numerosas opciones tecnológicas que hay que considerar: desde implementaciones inalámbricas, como el DLNA (Digital Living Network Alliance) o el AirPlay de Apple, hasta desarrollos que parten de la utilización de cableado con gran transferencia de datos para lograr alta definición (HDMI).

En definitiva, esta labor investigadora intenta interpretar y comprender los nuevos modelos relativos a las nuevas herramientas de carácter móvil que se utilizan en la proyección de contenidos en las aulas, para ello, esta investigación se apoya en dos pilares fundamentales, un marco teórico, en el que se analizan todos los conocimientos existentes sobre el objeto de estudio; y un trabajo de campo, en el que se recoge información de los principales instrumentos de carácter móvil que actualmente se utilizan para la proyección de contenidos.

Este estudio ofrece un retrato robot relativo a la proyección de cualquier tipo de contenido en el panorama educativo utilizando como fuente los distintos dispositivos móviles actuales, y brinda nuevas propuestas en las que convergen las nuevas tecnologías móviles aplicadas al objeto de estudio para lograr los objetivos competenciales.

\section{Nuevas Tecnologías en educación}

Existe una clara y estrecha relación entre los términos nuevas tecnologías y transmisión de conocimientos, entendiendo las nuevas tecnologías como material, medios y recursos que se utilizan en las clases para ayudar y mejorar la transmisión de saberes. Desde un libro, hasta la utilización de pizarras digitales, pasando por las aplicaciones personalizadas plataformadas en dispositivos móviles, todo ello son adapta- 
ciones tecnológicas aplicadas al mundo de la educación. El profesor Area Moreira (2009) defiende el término Tecnología Educativa "como una disciplina pedagógica".

El gran desarrollo de las nuevas tecnologías educativas, sufrido en la segunda mitad del siglo XX, no afecta significativamente a los métodos tradicionales de enseñanza, ya que estos, han encontrado su hueco, y han sabido adaptarse como complementos educativos. Los nuevos avances implantados en las aulas ofrecen una serie de ventajas que se pueden evidenciar según su usuario:

Desde el punto de vista del alumno las nuevas tecnologías aplicadas a la educación le permiten acceder a todos los recursos y prácticas de la materia docente que el profesor haya querido poner al alcance de sus alumnos, además, su disponibilidad es ilimitada y flexible, ya que toda la documentación está disponible las 24 horas del día en cualquier lugar, se evitan los costes en desplazamiento.

Por su parte, los profesores, con la aplicación de las nuevas tecnologías en las aulas, se logra una mayor motivación por parte de los alumnos y un constante feedback, el docente dispone de herramientas que consiguen una comunicación fluida entre alumnos y profesores. Gracias a las nuevas tecnologías aplicadas a la educación se logra un entorno de pruebas real para la realización de prácticas.

Pero también hay desventajas a la hora de implantar los avances tecnológicos en el mundo de la docencia, entre ellas se puede destacar por ejemplo la saturación por exceso de información, en la actualidad es muy complicado discriminar toda la información para conseguir encontrar documentación validad y de calidad.

El objetivo es superar todas estas barreras para conseguir una plena integración en las aulas para lograr que estos avances colaboren y faciliten la plena transmisión de conocimientos. Con esta implantación los roles de alumnos y profesores cambian completamente, el alumno necesita ser más participativo y colaborativo, mientras que los profesores tienen que dedicar tiempo en darle forma a los recursos tecnológicos que utilizarán y en una atención más personalizada al alumno. Además, los profesores necesitan actualizar constantemente sus materias y prácticas.

Por lo tanto, con la implantación de las nuevas tecnologías en las aulas se produce un cambio cultural, una transformación en las formas de trabajar. Todo ello requiere que sea fomentado por los actores implicados, hay que desarrollar la alfabetización tecnológica en las aulas.

La alfabetización tecnológica potencia y motiva la formación virtual, ofreciendo oportunidades de aprendizaje a lo largo de la vida pero se necesitan una planificación y contextualización de las actuaciones antes de comenzar cualquier proceso de alfabetización digital (Ortega Sánchez, 2006. Pág. 21).

Además, para lograr la integración tecnológica en las aulas se debe incentivar el trabajo constante con las nuevas tecnologías en todos los ámbitos de la sociedad.

La alfabetización no puede simplificarse en el conocimiento y manejo de las destrezas básicas lectoescritoras (hablar, escuchar, leer y escribir) en una sociedad 
actual. De ahí que sea preciso considerar la alfabetización en medios o audiovisual, ya que los aprendizajes de nuestra época son en clave audiovisual (Aguadez Gómez y Pérez Rodríguez, 2006. Pág. 69).

En este sentido aparecen las nuevas tecnologías móviles para tratar de adaptarse con elementos ya existentes, como la colocación de sistemas de proyección, para conseguir una mayor agilidad en el proceso educativo.

\section{Los dispositivos móviles como fuente en la proyección de contenidos en las aulas}

4.1 Una breve visión histórica del proyector y la integración de sus diferentes tecnologías

Ya se ha estudiado con anterioridad que los conceptos de tecnología y educación están estrechamente relacionados, la aparición de nuevos avances en la mayoría de los campos se ha traducido casi inmediatamente en su aplicación práctica en las aulas. Desde la invención de los tipos móviles de Guttemberg hasta el desarrollo de proyectores $4 \mathrm{~K}$. El primer invento relacionado con la proyección de contenidos en las aulas se remonta al siglo XVII, la linterna mágica de Athanasius Kircher, un artefacto que proyectaba imágenes dibujadas sobre vidrios. Posteriormente se desarrolló el episcopio, de principios del Siglo XX, era un proyector opaco que emitía una luz brillante que se proyectaba sobre un objeto no transparente.

Pero el gran impulsor de la proyección de contenidos para la instrucción fue el ejército norteamericano, que en 1945 utilizaba proyectores para la formación de sus soldados en las tácticas y misiones en el campo de batalla de la Segunda Guerra Mundial. Más adelante, en 1950 la empresa 3M, conocida por incentivar la investigación entre sus trabajadores consiguió que un investigador, Roger Appledorn, creara un artilugio para proyectar la escritura de una película transparente. De este modo se crearon los primero retroproyectores del mercado. El gran avance de esta herramienta se produjo en el momento en el que se desarrollaron las fotocopiadoras, ya que las notas manuscritas sobre transparencias no tuvieron mucho éxito por parecer informales.

Posteriormente, en los años 80 los docentes utilizaban proyectores para imágenes en movimiento, utilizando la tecnología CRT y posteriormente LCD.

Realmente, la implantación masiva de proyectores en las aulas se produce en la década de los 90, cuando aparecen tecnologías que mejoran sustancialmente la luminosidad y la resolución de los proyectores a un precio asequible. Actualmente se sigue innovando en este sentido, ya existen proyectores en alta definición, proyectores pequeños y potentes que facilitan su traslado o proyectores que son capaces de administrar vídeos en tres dimensiones. 
Hoy en día, la mayoría de las clases de todos los centros educativos cuentan con un sistema de proyección, aunque la proyección utilizando dispositivos móviles sigue siendo una asignatura pendiente, ya que estos espacios no están preparados.

Cuando se habla de sistemas de proyección, uno de los factores a tener en cuenta, y es casi tan importante que el sistema de proyección, es la fuente desde la que se quieren proyectar los contenidos, para este trabajo de investigación se quieren alcanzar conclusiones sobre sistemas de proyección utilizando tecnologías de carácter móvil.

La evolución en cuanto a los contenidos mostrados en los últimos 100 años también ha sido evidente, se ha pasado de la proyección de imágenes estáticas a la muestra de contenidos dinámicos, con movimientos, en ambientes con mucha luz, con una calidad tan alta que roza los límites de la visión humana.

En este sentido, aparece el término de resolución de imagen, su evolución ha sido evidente y ha recorrido el camino de forma paralela al resto de avances en la proyección de contenidos. La resolución está relacionada con la cantidad de información que se muestra en una proyección, cuanta más información (en píxeles) mayor resolución. Este avance ha logrado una mejora evidente en nitidez y se ha ganado en la muestra de detalles en los contenidos. Las principales resoluciones que conviven en la actualidad son:

- SVGA formada por 800 píxeles de ancho por 600 de alto.

- XGA formada por 1024 píxeles de ancho por 768 de alto.

- 720p formada por 1280 píxeles de ancho por 720 de alto.

- 1080 p formada por 1920 píxeles de ancho por 1080 de alto.

La realidad es que la resolución es uno de los factores que hay que tener en cuenta en la colocación de un proyector en un aula, pero existen otros factores que pueden determinar el éxito o fracaso, como puede ser su colocación, distancia proyector-pantalla, el tamaño de proyección la luminosidad natural del espacio, la potencia lumínica del proyector o la tecnología de proyección adoptada.

Hoy en día conviven dos tecnologías de proyección principales y alguna que trata de emerger:

Tecnología DLP (Digital Light Processing), desarrollado por la empresa norteamericana Texas Instruments y cuya línea principal se divide en los proyectores con un solo chip DMD (Digital Micromirror Device), y los proyectores que cuentan con tres de estos chips que generan la imagen en su interior. Las principales ventajas a la hora de utilizar esta tecnología es la buena reproducción de color, la gran capacidad de contrastar las imágenes mostradas, las dimensiones y el peso de los dispositivos de proyección, la larga vida lumínica de las lámparas y bajo coste. Respecto a las desventajas, hay que destacar que en algunos casos se crean efectos visuales que no se corresponden con la realidad que se quiere proyectar. 
Tecnología LCD, o también denominado sistema de pantalla de cristal líquido. Este es el más común y uno de los que predomina el mercado en la actualidad. Es la tecnología que gracias a su bajo coste ha estandarizado el uso de proyectores en las aulas de todo el mundo. La luz que emerge de la lámpara de proyección se divide en tres y se pasa por tres paneles de cristal líquido, uno para cada color (rojo, verde y azul). Esta luz se vuelve a unir y recompone la imagen. La mayor ventaja de este sistema de proyección es la fiabilidad y sus mayores inconvenientes son, la posible pixelación de los contenidos y los denominados píxeles muertos.

Otras tecnologías. En la actualidad se desarrollan nuevas tecnologías que tratan de superar las existentes, como los proyectores D-ILA (Direct-drive Image Light Amplifier), o la tecnología 3D.

\subsection{El proyector como herramienta fundamental en los procesos educativos}

El rendimiento de los estudiantes y la búsqueda de la eficiencia docente son los principales objetivos que se buscan a la hora de implantar las nuevas tecnologías en las aulas. En este sentido, los centros educativos invierten fondos en la adaptación de sus espacios docentes a los nuevos avances. Según muestra un estudio referenciado por la página web Nido IT (Kitch, 2010), en el que se muestran los beneficios de la utilización de los proyectores en las aulas, "el 98\% de los encuestados afirma que los proyectores proporcionan una mejora significativa en la atención de los estudiantes, el 94\% señaló que se mejoraba significativamente la calidad de las presentaciones y su edición, el 94\% afirmó que con el uso de estos dispositivos se consigue una mayor facilidad en la presentación de la información. Para el 90\% existe una mejoría significativa de la comprensión de la información. El 80\% aseguró que se mejoraba de forma sustancial la retención de la información. Para el $74 \%$ de los encuestados, se ahorra tiempo total a la hora de impartir docencia. El 62\% asevera que la instalación de estos dispositivos reportan un ahorro significativo de costes".

La mejora que aporta la instalación de un proyector en un aula evidente en una transmisión de conocimientos más efectiva. Gracias a este elemento, la formación se ha ce más visual y dinámica, el docente puede mostrar cualquier contenido sin ningún tipo de restricción. El acceso a cualquier contenido audiovisual por parte de todos los integrantes de un curso facilita notablemente la comprensión de los contenidos, el acceso a la información que quiere mostrar el docente se evidencia y desaparece otro tipo de contenido, que antaño era entregado en forma de fotocopias.

Pero, el gran avance no es sólo la mejora en la proyección de contenidos, o la instalación de proyectores en las aulas, o el acceso a internet de los ordenadores utilizados para la proyección, o el manejo masivo de los dispositivos móviles por parte de todos los actores que intervienen en el proceso educativo, o la creación de entornos adaptados a la formación, el gran punto de inflexión se encuentra en la combinación de todos ellos con un único objetivo: La transmisión de saberes.

El éxito de la implantación de sistemas de proyección en las aulas está suficientemente abalado, ya que, no importando el tipo conocimientos que se quiera transmitir 
esta es una herramienta transversal que por ejemplo está instalado en universidades, autoescuelas, colegios, escuelas de negocio, etc.

Aunque en apariencia la implantación de este tipo de dispositivos en las clases sea ventajosa, existen algunas barreras que hay que superar, la principal es la inversión económica que hay que realizar, ya que en muchos casos hay que adaptar el aula para mejorar las condiciones de proyección, y por otro lado, hay que realizar una instalación de un equipo nuevo con un coste incial y otro de mantenimiento.

Otra barrera que hay que tener en cuenta es el denominado miedo al cambio que algunos docentes sufren, ya que aferrándose a los medios tradicionales de transmisión de conocimientos, son incapaces de asimilar al mismo tiempo que sus alumnos la aparición de nuevas tecnologías y avances en las aulas. En este sentido, es óptima la formación que se debe impartir a los docentes para que sean capaces de integrar todas las ventajas de las nuevas tecnologías en las aulas. Para ello, los profesores deben tener una actitud receptiva y encaminada a la mejora educativa.

La razón de este estudio parte de otra limitación importante que aparece con la instalación de proyectores en las aulas, es la limitación de movimiento a la que el profesor es obligado. Los docentes se pueden sentir fijados a su mesa por un cable que conecta el ordenador con el proyector. Por ello, este estudio de investigación trata de mostrar las tecnologías y desarrollos que pueden desatar al docente de su mesa para que puedan moverse libremente por las aulas sin que empeores los contenidos proyectados.

4.3 Posibilidades técnicas en para la proyección de contenidos mediante dispositivos móviles en las aulas

Para tratar de solventar la movilidad de los profesores en las aulas emergen una serie de tecnologías que tratan de eliminar los cables que unen los ordenadores con los proyectores, el problema principal es la gran cantidad de información que requiere la transferencia de imágenes en movimiento. En paralelo, como ya se ha nombrado con anterioridad, surgen dispositivos móviles con un gran potencial que pueden ser herramientas ideales para el trabajo en las clases.

Hay que recordar que en su inicio, los dispositivos móviles utilizados en las aulas, supusieron un claro perjuicio para la labor educativa por culpa de las continuas interrupciones que provocaban. Que incluso se llegó a legislar la limitación de uso. La Comunidad de Madrid limitó la utilización del teléfono móvil en las aulas, expresado en el DECRETO 15/2007, de 19 de abril, por el que se establece el marco regulador de la convivencia en los centros docentes de la Comunidad de Madrid. Aunque con posterioridad se ha tratado de acercar esta realidad tecnológica a su uso docente surgiendo términos como el M-Learnig que sólo ha sido posible con la aparición de los teléfonos y tabletas de última generación.

Además, todo ello ha sido posible gracias a la estandarización y desarrollo exponencial de estos artefactos. Según el informe Gartner (2012) en el cuarto trimestre 
de 2011 las ventas de Smartphones sufrieron un incremento de un 47,3\% respecto al mismo periodo del año anterior. La compañía Apple ha duplicado las ventas y Samsung incrementa su cuota de forma más moderada.

Quizá el momento actual del objeto de estudio sea el de estabilización del mercado, siempre que aparece un punto de inflexión en las nuevas tecnologías, emergen tecnologías desarrolladas por diferentes empresas que quieren copar el mercado con sus ideas y creaciones. En función de la tecnología utilizada tecnología se perciben dos tipos de conexiones entre dispositivos móviles y proyectores en las aulas:

Conexión mediante cable. En un principio se apostó por este tipo de transferencia de datos. Es un sistema fiable, ya que la pérdida de datos es inexistente. Pero esta forma de proyectar elimina el carácter móvil del dispositivo y no soluciona la premisa de buscar una mayor libertad de movimiento para el profesor. Utilizando este sistema se puede hacer una división según la calidad de la emisión de contenidos:

- Video Graphics Array (VGA). Conexión y calidad gráfica tradicional de video entre un ordenador y un proyector. Su principal éxito ha sido la estandarización del mercado en la conexión de video en equipación informática. Según la fuente móvil de proyección, en la actualidad encontramos diferentes sistemas:

- Dispositivos IOS. Para este tipo de dispositivos existen en el mercado conectores que transforman la salida de imagen en una salida VGA estándar.

- Dispositivos Android. Para este tipo de dispositivos también se pueden adquirir gran cantidad de adaptadores que transforman su salida a una conexión VGA, pero su estandarización no está conseguida, ya que se distribuyen en función de su fabricante.

- Otros Dispositivos. En este caso cada desarrollador y fabricante ha creado sus conectores.

- High-Definition Multimedia Interface (HDMI). Este tipo de calidad y conexión se está apoderando del mercado por la ventajosa calidad de imagen que ofrece. Cada fabricante y desarrollador ha creado sus conectores, estandarizando la entrada en el conector HDMI.

Conexión inalámbrica. La forma de administrar y gestionar archivos se ha transformado en los últimos años, profesores y alumnos almacenan y comparten sus apuntes y prácticas en la red y acceden a ellos a través de sus dispositivos móviles. Por esta razón, los docentes buscan la forma de poder proyectar los contenidos de sus tabletas en las aulas, y para no limitar sus movimientos buscan soluciones inalámbricas. Fabricantes y desarrolladores ofrecen soluciones inalámbricas para la transmisión de información entre dispositivos móviles y proyectores. Entre las principales se pueden citar:

Digital Living Network Alliance (DLNA). Fue creada por un conjunto de fabricantes con la dirección de Sony. Este tipo de tecnología trata de crear redes internas 
utilizando los dispositivos de conexión inalámbrica de los dispositivos para compartir archivos y poder reproducirlos en cualquier dispositivo compatible.

AirPlay. Por su parte, Apple desarrolló paralelamente a la tecnología DLNA, AirPlay, en sus inicio AirTunes, para ofrecer una solución inalámbrica a sus clientes. En la actualidad esta tecnología es la más avanzada, ya que permite clonar la pantalla del dispositivos haciendo una función de espejo.

Otras soluciones. El resto de fabricantes y desarrolladores han tratado de buscar soluciones en este sentido, por ejemplo, la empresa Epson ofrece la tecnología iProjection que conecta un dispositivo móvil con un proyector de forma inalámbrica para la reproducción de todo tipo de contenidos.

En la actualidad existen tecnologías capaces de conectar con un proyector de forma inalámbrica utilizando las distintas soluciones que ofrecen desarrolladores y fabricantes.

\section{Conclusiones}

Tras la realización de un estudio exhaustivo de las diferentes opciones de conexión entre los denominados dispositivos móviles y los dispositivos de proyección en las aulas se puede llegar a las siguientes conclusiones:

- Los profesores y alumnos son conocedores de los avances en las nuevas tecnologías y tratan de aplicarlas a los procesos educativos para lograr una transmisión de saberes más efectiva. Con los dispositivos móviles estos actores disponen de herramientas potentes que están conectadas a todos los contenidos de las materias que les ocupan y son capaces de interrelacionarse de una forma fluida utilizando aplicaciones integradoras y colaborativas.

- Los proyectores en las aulas se han convertido en un elemento casi indispensable, ya que logra una mayor eficiencia en la transmisión de conocimientos. En la actualidad el objetivo es lograr la mayor conectividad posible entre estos dispositivos y los dispositivos que emergen en el mercado de las tecnologías.

- Una de las principales barrera a la hora de proyectar los contenidos de los dispositivos móviles en las aulas, es la utilización de conectores cableados, ya que limitan la libertad de movimiento del profesor y anulan el carácter móvil de sus dispositivos. Actualmente existen tecnologías que hacen posible una conexión inalámbrica de calidad para la emisión de contenidos en las aulas utilizando tabletas o smartphones. 


\section{Bibliografía}

\subsection{Libros:}

CABERO, Julio (2001): Tecnología Educativa. Diseño, uti-lización y evaluación de medios de enseñanza. Paid-ós. Barcelona.

DANIEL, John (1996): Megauniversities and Knowledge Media. Kogan Page, London.

HARGREAVES, Andy (2003): Enseñar en la Sociedad del Conocimiento. Octaedro. Barcelona.

FRANCO, Jorge (2008): Educación y tecnología: Solución radical. Siglo XXI. Mexico.

KOZMAN, Robert (2003): Technology, Innovation, and Educational Change. A Global Perspective. ISTE. Washington.

MINISTERIO DE EDUCACIÓN Y CIENCIA (2005): Aplicaciones educativas de las Tecnologías de la Información y la Comunicación. Secretaría General Técnica. Madrid.

SALINAS, Jesús; AGUADED, José Ignacio; CABERO, Ju $\neg$ lio (coords.) (2004): Tecnologías para la educación. Diseño, producción y evaluación de medios para la formación docente. Alianza. Madrid.

SANCHO, Juana María (2006): Tecnologías para transfor $\neg$ mar la educación. Akal. Madrid.

6.2 Capítulos o artículos en libros o revistas en papel:

AGUADEZ GÓMEZ, J. I. y PÉREZ RODRÍGUEZ, M. A. (2006): La educación en medios de comunicación como contexto educativo en un mundo globalizado, en CABERO ALMENARA. (Coord.) Nuevas tecnologías aplicadas a la educación. Madrid. McGraw-Hill.

6.3 Artículos en publicaciones web:

AREA MOREIRA, Manuel (2009): Introducción a la Tecnología Educativa. Disponible en: http://bibliotecadigital.org/bitstream/001/415/5/Introducci $\% C 3 \%$ B3n $\% 20$ a\%201a\%20tecnolog\%C3\%ADa\%20educativa.pdf. [10-08-2013].

GARTNER (2012): Worldwide Smartphone Sales Soared in Fourth Quarter of 2011 With 47 Percent Growth. Disponible en: http://www.gartner.com/it/page. jsp?id=1924314 [24-08-2013].

ORTEGA SÁNCHEZ, I. (2009): La Alfabetización tecnológica”. En ORTEGA SÁNCHEZ, I. y FERRÁS SEXTO, C. (Coord.) Alfabetización Tecnológica y el desarrollo regional en Revista Electrónica Teoría de la Educación: Educación y Cultura en la Sociedad de la Información. Vol. 10, $\mathrm{n}^{\mathbf{0}} 2$. Universidad de Salamanca. http://www.usal.es/ teoriaeducacion/rev_numero_10_02/n10_02_ ortega_sanchez.pdf.[13-08-2013].

KITCH, Derwin (2010): Data Projectors, en NidoIT. Disponible en: http://nidoit. wikispaces.com/Data+Projectors [25-08-2013]. 


\section{El autor}

Héctor Molina García, Doctor en Periodismo por la Universidad Complutense de Madrid con la calificación de Sobresaliente "Cum Laude", desarrolla su actividad profesional en el campo de las Nuevas Tecnologías de la Información desde el Departamento de Tecnologías de la Universidad Francisco de Vitoria y desde las facultades de Periodismo y Magisterio de dicho centro educativo. Coordinador e integrante de proyectos de investigación relacionados con sus áreas de estudio imparte asignaturas pertenecientes a los campos tecnológicos. Destacan sus publicaciones en revistas de carácter científico sobre temas relacionados con las nuevas tecnologías y la educación. 